The writer's assertion that not all doctors have time, inclination or aptitude to adopt models of patientdoctor interaction as expounded by psychologists such as Balint is counter to the recommendations of the General Medical Council quoted in the preface.

There is scant reference to the many ethical problems generated by poor communication. While there is brief discussion of confidentiality and informed consent, difficult areas which so concern students such as breaking bad news, how to request a postmortem, the switching off of a respirator, how to deal with discussions on euthanasia, and 'not for resuscitation orders' receive little or no mention. AIDS, the greatest challenge to communication skills and one which is compounded by ethical problems, receives little more than a single page.

However, there are some gems; the sections on talking to the elderly, and to the dying and especially that on talking to adolescents, are a welcome addition to the literature.

F M HULL fasmine Cottage, Hampton Lucy, Warwick CV35 8BE

\section{Race relations: code of practice in primary health care services}

\author{
Commission for Racial Equality, \\ London, CRE, 1992, 51 pages, \\ £1.50.
}

The principle of justice, however poorly understood, is generally accepted as an important ethical precept of health care staff. Why then is it necessary for the Commission for Racial Equality to publish a code of practice on race relations in primary health care services? This should, 18 years after the relevant legislation was enacted, in the form of the Race Relations Act, 1976, be part of the culture of the health care services and require little new writing.

The principles of equity and justice are not referred to in the code; the legislation is. It is a sad reflection on the current state of health care ethics if fear of legal consequences produces more action than the underlying ethical principles. The sad fact is that discrimination within the health service as an employer is commonplace with, for example, overseas doctors poorly represented amongst consultants ( 14 per cent in 1975 rising to 17 per cent in 1981), and discrimination in relation to the provision of care continues.

The cases quoted by the CRE are the tip of the iceberg. Most discrimination is institutionalised and results from a lack of understanding of the needs of ethnic minority patients, a lack of resources to deal with the inequities which arise and in some circumstances a lack of interest. The CRE discusses indirect discrimination in detail, and later provides examples where positive action can help. But it does not address the issue of resources. Who will pay for professional interpreters in primary care, and should they replace staff providing hands-on care at a time when money for staffing of primary care is increasingly squeezed?

The reader will first be horrified by the examples, mostly based on real cases, of discrimination and victimisation. But that is to miss the more important element - the requirement to act positively to prevent inequalities, to gather information on service needs, accessibility and provision, and to monitor health services on the basis of equity as well as cost/ benefits. These are all, of course, requirements in addition to those of the NHS and Community Care Act, and to the raft of further work required by the Patient's Charter and the Health of the Nation. But action in primary care is possible: in particular, audit of service access to ethnic minority groups, training of staff on anti-discrimination measures, and a concerted move under the health promotion action to fund the training and employment of interpreters for the primary health care setting.

More fundamentally, the cases described by the CRE clearly demonstrate that the lack of teaching on ethics in many medical schools has practical consequences, and they provide more evidence of the need for formal required training at undergraduate level and within continuing medical education.

VIVIENNE NATHANSON Scottish Secretary, British Medical Association, 3 Hill Place Edinburgh
Counselling in general practice

Edited by Roslyn Corney and Rachel Jenkins, London, Routledge, 1993, 119 pages, £9.99.

In this book Roslyn Corney, Senior Lecturer at the Institute of Psychiatry and in the Centre for Health Service Studies at the University of Kent, and Rachel Jenkins, Principal Medical Officer of the Mental Health, Elderly and Disability Unit at the Department of Health, present a series of papers on the subject of counselling in general practice. The book is aimed at a readership of general practitioners, counsellors and other members of the primary care team. At the same time a number of the issues examined in the various papers would be of interest to any reader wishing to gain greater insight into, and understanding of, counselling as a function, not just within general practice.

The ten papers by a wide range of contributors, collected together in this book, cover a comprehensive range of issues relevant to counselling in general practice. A clear and conect cise account is provided of the need? for counselling in general practice in order to meet the requirements presented by the high level of psychological and psychiatric disorders diagnosed in primary care. In her paper, Nancy Rowland explains what, precisely, counselling is, who it is for and how it differs from a consultation with the GP or any other member of the primary care team. She establishes that counselling is an ethical task, rooted in a code of ethics and practice, which enables the counsellor to create a therapeutic alliance with the client in order to help the client to discover a way to work towards the resolution of problems and find a way of living more resourcefully. In other papers the book presents an analysis of the effectiveness of counselling in general practice and examines methods of evaluation of such counselling. The book presents illuminating papers on the practical issues regarding how to find a well qualified and experienced counsellor and how to go about setting up a counselling attachment. A range of associated issues are discussed in detail and two existing coun-selling attachments are presented.

Of particular interest is a paper by Rachel Jenkins and Raanan Gillon on the ethics of counselling. This is an 